\title{
Tratamento conservador em reabilitação oral através da interação entre Odontologia Restauradora e Prótese Dentária
}

Conservative treatment in oral rehabilitation through the interaction between Restorative Dentistry and Dental Prosthesis Tratamiento conservador en rehabilitación oral a través de la interacción entre Odontología Restauradora y Prótesis Dental Cecília Alves de SOUSA ${ }^{1}$

Keith Murieli Ferreira de MAGALHÃES

Gustavo Antônio Correa MOMESSO ${ }^{2}$

Lara Maria Bueno ESTEVES ${ }^{3}$

Isis Almela Endo HOSHINO

Leonardo Perez FAVERANI ${ }^{4}$

Paulo Henrique dos SANTOS ${ }^{5}$

Wirley Gonçalves ASSUNÇÃO ${ }^{5}$

${ }^{1}$ Postgraduate student, Department of Dental Materials and Prosthodontic, São Paulo State University (UNESP) Araçatuba School of Dentistry, 16015-050 Araçatuba - SP, Brazil ${ }^{2}$ Postgraduate student, Department of Surgery and Integrated Clinic, São Paulo State University (UNESP) Araçatuba School of Dentistry, 16015-050 Araçatuba - SP, Brazil

${ }^{3}$ Postgraduate student, Department of Restorative Dentistry, São Paulo State University (UNESP) Araçatuba School of Dentistry, 16015-050 Araçatuba - SP, Brazil ${ }^{4}$ Professor, Department of Surgery and Integrated Clinic, São Paulo State University (UNESP) Araçatuba School of Dentistry, 16015-050 Araçatuba - SP, Brazil

${ }^{5}$ Associate professor, Department of Dental Materials and Prosthodontic, São Paulo State University (UNESP) Araçatuba School of Dentistry, 16015-050 Araçatuba - SP, Brazil

\section{Resumo}

Este estudo teve como objetivo, apresentar por meio de um relato de caso a interação entre odontologia restauradora e prótese dentária, orientando ao clínico uma opção de conduta clínica minimamente invasiva no tratamento de reabilitação oral. Paciente do sexo masculino, 49 anos, com queixa principal de mobilidade nos dentes anteriores superiores que sustentavam uma prótese fixa e insatisfação estética do sorriso. O exame clínico foi realizado e constatou-se situação periodontal comprometida de todos os dentes superiores, periodontite com grau 2 de mobilidade e reabsorção óssea vertical severa nos dentes 31, 32, 37 e 42, ausência dos elementos 36 e 41 e mobilidade grau 2 nos demais dentes inferiores em função da má oclusão. Foi planejada extração dentária dos dentes superiores, seguida de levantamento de seio bilateral e instalação de seis implantes para prótese do tipo protocolo de Banemärk. No arco inferior, raspagem e alisamento radicular, extração dos dentes 31, 32, 37 42, instalação de implantes nas regiões dos dentes 32, 36 e 42 e instalação de prótese implantosuportada. Considerando a necessidade de reestabelecer a oclusão, a condição periodontal comprometida dos dentes inferiores posteriores e a necessidade de melhorias estéticas, foi optado pela realização de restaurações em resina composta do tipo BulkFill ${ }^{\circledR}$ através da técnica do carimbo modificado, reproduzindo a anatomia oclusal dos elementos. Dessa forma, podemos concluir que a interação da odontologia restauradora e a prótese dentária pode ser uma alternativa interessante para casos em que há necessidade de tratamento reabilitador conservador, visando obter resultado de excelência na reabilitação oral.

Descritores: Prótese Dentária; Prótese Dentária Fixada por Implante; Reabilitação Bucal.

\section{Abstract}

This study aimed to present, through a case report, the interaction between restorative dentistry and dental prosthesis, guiding the clinician an option of minimally invasive clinical conduct in the treatment of oral rehabilitation. Male patient, 49 years old, with main complaint of mobility in the upper anterior teeth that supported a fixed prosthesis and aesthetic dissatisfaction of the smile. The clinical examination was carried out and a periodontal condition of all upper teeth was found, periodontitis with grade 2 mobility and severe vertical bone resorption in teeth 31, 32, 37 and 42, absence of elements 36 and 41 and grade 2 mobility in the teeth. other lower teeth due to malocclusion. Dental extraction of the upper teeth was planned, followed by a bilateral sinus lift and the installation of six implants for the prosthesis of the Banemärk protocol type. In the lower arch, scaling and root planing, extraction of teeth $31,32,3742$, installation of implants in the regions of teeth 32, 36 and 42 and installation of implant-supported prosthesis. Considering the need to reestablish the occlusion, the compromised periodontal condition of the posterior lower teeth and the need for aesthetic improvements, it was decided to perform restorations in composite resin of the BulkFill@ type through the modified stamp technique, reproducing the occlusal anatomy of the elements. Thus, we can conclude that the interaction of restorative dentistry and dental prosthesis can be an interesting alternative for cases in which there is a need for conservative rehabilitation treatment, aiming to obtain excellent results in oral rehabilitation.

Descriptors: Dental Prosthesis; Dental Prosthesis, Implant-Supported; Mouth Rehabilitation.

\section{Resumen}

Este estudio tuvo como objetivo presentar, a través de un caso clínico, la interacción entre la odontología restauradora y la prótesis dental, orientando al clínico una opción de conducta clínica mínimamente invasiva en el tratamiento de la rehabilitación oral. Paciente de sexo masculino, 49 años, con principal queja de movilidad en los dientes anteriores superiores que soportaban una prótesis fija e insatisfacción estética de la sonrisa. Se realizó el examen clínico y se encontró condición periodontal de todos los dientes superiores, periodontitis con movilidad grado 2 y reabsorción ósea vertical severa en los dientes 31, 32 , 37 y 42 , ausencia de elementos 36 y 41 y movilidad grado 2 en los dientes .Otros dientes inferiores por maloclusión. Se planificó la extracción dental de los dientes superiores, seguida de un levantamiento de seno bilateral y la instalación de seis implantes para la prótesis tipo protocolo Banemärk. En la arcada inferior, raspado y alisado radicular, extracción de los dientes 31, 32, 37 42, instalación de implantes en las regiones de los dientes 32 , 36 y 42 e instalación de prótesis implantosoportada. Considerando la necesidad de restablecer la oclusión, el comprometido estado periodontal de los dientes posteriores inferiores y la necesidad de mejoras estéticas, se decidió realizar restauraciones en resina compuesta del tipo BulkFill@ mediante la técnica de estampa modificada, reproduciendo la anatomía oclusal de la elementos. Así, podemos concluir que la interacción de la odontología restauradora y la prótesis dental puede ser una alternativa interesante para los casos en los que existe la necesidad de un tratamiento rehabilitador conservador, con el objetivo de obtener excelentes resultados en la rehabilitación oral.

Descriptores: Prótesis Dental; Prótesis Dental de Soporte Implantado; Rehabilitación Bucal.

INTRODUÇÃO

A correta manutenção da saúde bucal, função mastigatória e estética são fundamentos importantes na odontologia. Durante a evolução da reabilitação oral, a implantodontia permitiu o restabelecimento da função em pacientes parcialmente ou completamente edêntulos, sendo uma opção de tratamento viável em conjunto com a reabilitação por meio de próteses dentárias ${ }^{1}$. Durante a reabilitação destes pacientes é possível observar que com as perdas dentárias, ocorre a extrusão dos dentes adjacentes à área desdentada e com o 
desgaste dentário excessivo devido sobrecarga mastigatória, pode ocorrer perda da dimensão vertical, alteração do terço inferior da face e prejuízos estéticos. Quando isto ocorre, o nível de dificuldade no tratamento aumenta e torna-se ainda mais necessário seguir a estratégia terapêutica mais apropriada e rigorosa para 0 caso proposto ${ }^{1,2}$.

A ideia de aumentar a dimensão vertical de oclusão foi descrita por Dhal ${ }^{3,4}$, visando melhora na função, na estética, redução da hipersensibilidade térmica e diminuindo a possibilidades de abrasão da estrutura dentária ${ }^{5,6}$. Neste contexto, várias técnicas usando restaurações diretas ou indiretas de compósitos em resina, cerâmica ou materiais híbridos foram descritas para a reconstrução de dentes desgastados e o restabelecimento da oclusão $^{7-13}$. Os conceitos tradicionalmente reconstrutivos incluem principalmente coroas de porcelana fundida ao metal ou sobreposições de cerâmica. Entretanto, o tratamento por meio de cerâmicas é invasivo, prolongado e de custo mais alto para o paciente ${ }^{7}$.

Hoje, o compósito de resina direta tornou-se um material estabelecido para restauração oral minimamente invasiva dos dentes posteriores com desgaste erosivo ${ }^{14}$. Porém, reconstruir a mão livre uma morfologia dentária com a finalidade de obter uma oclusão funcional é um processo demorado e minucioso. Visando solucionar este problema, foi desenvolvido novo método de reconstrução por mordida vertical posterior de dentições desgastadas denominado técnica de carimbo oclusal, utilizando gabaritos de silicone ${ }^{14}$. Estes são posicionados na vestibular e lingual de cada dente, que são preenchidos com resina composta para transferir a restauração planejada e futura na forma do enceramento da situação extra para a intraoral ${ }^{14,15}$.

Este estudo teve como objetivo, apresentar por meio de um relato de caso a interação entre odontologia restauradora e prótese dentária, orientando ao clínico uma opção de conduta clínica minimamente invasiva no tratamento de reabilitação oral.

CASO CLÍNICO

Paciente do sexo masculino, 49 anos, foi encaminhado para a equipe de prótese da FOAUNESP com queixa de mobilidade em dentes anteriores superiores que sustentavam uma prótese fixa de estética comprometida e insatisfação estéticas do arco inferior (Figura 1A). O exame clínico foi realizado e constatou-se uma situação periodontal comprometida em todos os dentes superiores, periodontite com grau 2 de mobilidade nos dentes remanescentes inferiores e ausência dos elementos 36 e 41 . Assim, a conduta cirúrgica foi de extração de todos os dentes superiores, antrotomia e deslocamento da membrana do seio maxilar para levantamento de seio bilateral, seguido da instalação de 6 implantes para prótese protocolo de Branemärk (Figura 1B). Foi realizado por meio do acesso de Cadwell-Luc na parede do seio maxilar seguido da antrotomia da mambrana sinusal. O biomaterial selecionado foi osso bovino particulado (Criteria ${ }^{\circledR}$-Lumina-Bone, São Carlos, São Paulo, Brasil), preenchendo a região posterior do seio (Figura 1B). A seguir, foi realizada a fresagem subsequente para a instalação dos implantes no rebordo até atingir $13 \mathrm{~mm}$ de profundidade e, em seguida, foi instalado implantes de $4,1 \times$ $11 \mathrm{~mm}$ e na região anterior implantes de $3,75 \times$ 13 mm (Conexão Sistema de Prótese ${ }^{\circledR}$, Arujá, São Paulo, Brasil), seguido do preenchimento completo do seio maxilar com o biomaterial. Ao final desta etapa, uma membrana de colágeno reabsorvível $\quad$ (Criteria ${ }^{\circledR}$-Lumina-Coat, São Carlos, São Paulo, Brasil) foi posicionada cobrindo a antrotomia e foi realizada sutura completa da área cirúrgica em ambos os lados com fio de seda 4-0 (Figura 1C). No arco inferior, raspagem e alisamento radicular, extração dos dentes $31,32,37$ e 42, instalação de implantes nas regiões dos dentes $32(3,75 \times$ $13 \mathrm{~mm}), 36(4,1 \times 11 \mathrm{~mm})$ e $42(3,75 \times 13 \mathrm{~mm})$ (Conexão Sistema de Prótese ${ }^{\circledR}$, Arujá, São Paulo, Brasil) e instalação de prótese implantosuportada em e-max (IPS e.max CAD Ivoclar Vivadent ${ }^{\circledR}$ ) e devido à perda de altura gengiva, foram confeccionadas gengiva artificial cerâmica em e-max (IPS e.max CAD - Ivoclar Vivadent $^{\circledR}$ ) (Figura 1D-H).

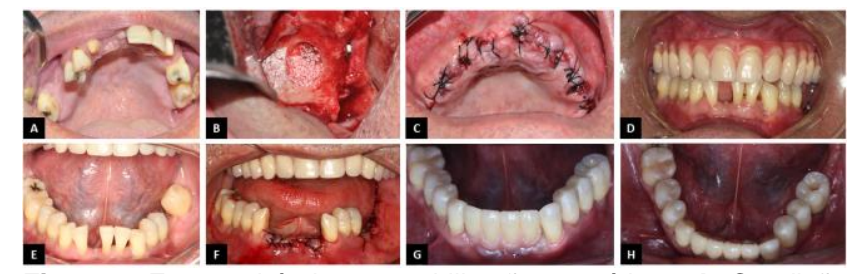

Figura 1: Etapas cirúrgicas e reabilitações protéticas. A: Condição inicial do arco superior. B: Alvéolos em exposição após extração dos elementos superiores, levantamento de seio maxilar com osso bovino particulado preenchendo o seio e implante instalado na região no mesmo momento cirúrgico. C: Sutura do arco superior após levantamento de seio maxilar e instalação de 6 implantes. D: Instalação da prótese protocolo de Branemärk após o período de osseointegração. E: Condição inicial do arco inferior. F: Sutura após extração dos elementos 37, 31, 32 e 42 e instalação de implantes na região dos elementos 32, 42 e 37. G: Próteses implanto suportadas em e-max na região anterior com gengiva artificial. H: Prótese unitária em e-max na região do elemento 37.

Além disso, considerando a condição periodontal desfavorável dos dentes remanescentes inferiores e a necessidade de 
melhoria estética, foi optado a restauração completa em resina composta através da técnica do carimbo modificado a partir de enceramento realizado e obtenção de moldeiras que reproduzem a anatomia oclusal. Após enceramento diagnóstico foi confeccionado matrizes de carimbo oclusal modificado com placa de acrílico de $1 \mathrm{~mm}$. Foi realizado profilaxia e preparo da estrutura dental para restauração com resina composta do tipo BulkFill $^{\Theta^{3}}$ (Filltech $^{\text {TM }}$ One Bulk Fill ${ }^{\circledR}$, 3M ESPE Dental Products $^{\mathrm{TM}}$, St. Paul, MN, USA) segundo protocolo recomendado pelo fabricante (Figura 2A-D).

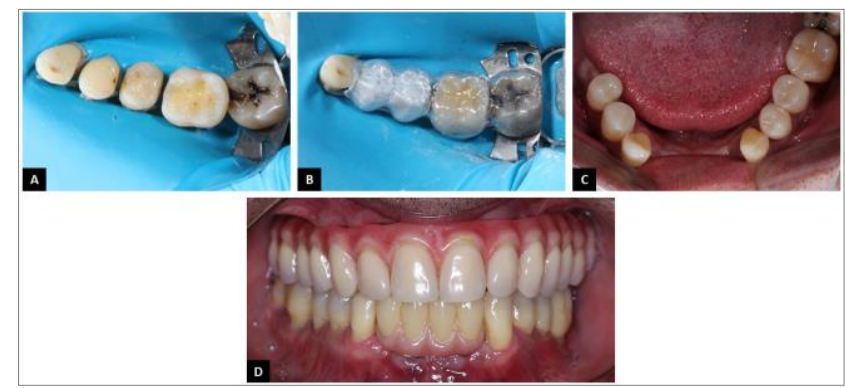

Figure 2: Restaurações dos elementos dentários inferiores em resina composta. A: Situação inicial dos dentes a serem restaurados com os pontos de contato rompidos em isolamento absoluto. B: Placa de acrílico em posição para execução da técnica do carimbo oclusal. C: Restaurações de resina composta finalizadas. D: Condição final após a reabilitação oral.

DISCUSSÃO

Todo procedimento de reabilitação oral deve apresentar um planejamento adequado e etapas a serem desenvolvidas, visando um sucesso em longo prazo. Além disso, os planos de tratamento devem considerar a perspectiva do paciente, antecedentes socioeconômicos, gravidade do distúrbio oclusal e condições bucais.

Pacientes que apresentam uma grande reabsorção óssea vertical em região posterior de maxila apresentam limitações anatômicas à instalação de implantes, como a proximidade do seio maxilar. A elevação do seio maxilar é uma técnica muito bem descrita na literatura. De acordo com o caso executado, a técnica de instalação de implantes simultânea a elevação do seio maxilar é benéfica para o paciente, uma vez que diminui o tempo operatório, torna a execução da prótese mais rápida, reduz a morbidade dos implantes e acelera o tempo de tratamento. Em relação a instalação dos implantes e a prótese protocolo permitiu ao paciente reabilitação adequada, uma vez que se não fosse por isso seria usado uma prótese total convencional ${ }^{16}$.

Este caso clínico relatou a devolução da anatomia dental e dimensão vertical de oclusão através da correlação entre compósitos em resina e a instalação das coroas sobre implante ${ }^{16,17}$. É sabido que o uso de restaurações cerâmicas apresenta excelentes resultados como coroas fixas em dentes unitários, além de apresentarem alta previsibilidade de tratamento, alta lisura superficial e baixa porosidade; principalmente, quando comparado à resina composta. Entretanto, no caso em questão, foi optado o uso de resina composta, em virtude da deficiência periodontal que não permitiria a garantia de previsibilidade alta no tratamento com cerâmicas. Sendo assim, a reabilitação nos dentes posteriores, a resina composta foi escolhida, pois o paciente possuía esmalte suficiente, o que garantiria adesão adequada do material ao dente e também pelo menor custo do tratamento em comparação às restaurações cerâmicas $^{16-18}$.

As indicações de resina composta aumentaram ao longo dos anos ${ }^{19}$, no entanto, estudos que relatam reabilitação completa da oclusão com restaurações diretas de resina composta são raros ${ }^{14} \mathrm{~A}$ principal vantagem de associar essa técnica à resina bulk-fill é realizar uma reabilitação satisfatória de maneira simples, bem como a boa camuflagem de cores do material restaurador, 0 que mostra resultados estéticos satisfatórios ${ }^{20}$.

A resina Bulk-fill ${ }^{\circledR}$ apresenta modificações em suas composições, como novos monômeros e mudanças em suas concentrações, adição de novos fotoiniciadores, aumento da translucidez e diminuição ou alteração de seu conteúdo inorgânico. Estas modificações permitiram sua aplicação nas espessuras de 4 a $5 \mathrm{~mm}$, diminuindo assim a tempo gasto no procedimento restaurador. Finalmente, a técnica restauradora direta descrita pode ser considerada em termos de relação custo-benefício. Sabe-se que a taxa média anual de falhas para restaurações posteriores compostas e cerâmicas não difere consideravelmente embora esses dados tenham sido coletados principalmente de restaurações menos extensas do que o caso apresentado ${ }^{20}$. Como a união com material compósito é um conceito conservador para restaurar uma dentição desgastada, o intuito deste caso é fazer com que o leitor considere o uso de abordagens adesivas como uma solução aceitável em médio prazo no tratamento de dentes desgastados severamente e devolução da dimensão vertical de oclusão juntamente com a instalação de implantes dentários e confecção de próteses sob implantes.

CONCLUSÃO

Dessa forma, podemos concluir que a 
interação da odontologia restauradora e a prótese dentária pode ser uma alternativa interessante para casos em que há necessidade de tratamento reabilitador conservador, visando obter resultado de excelência na reabilitação oral.

\section{REFERÊNCIAS}

1. Attin T, Filli $T$, Imfeld $C$, Schmidlin PR. Composite vertical bite reconstructions in eroded dentitions after 5.5 years: a case series. J Oral Rehabil. 2012;39(1):73-9.

2. Johansson A, Johansson AK, Omar $R$, Carlsson GE. Rehabilitation of the worn dentition. J Oral Rehabil. 2008;35(7):548-66.

3. Assaf C, Fahd JC, Sabbagh J. Four-Year Follow-up of Increased Vertical Dimension of Occlusion using Resin Composites. J Int Soc Prev Community Dent. 2018;8(5):469-74.

4. Dahl BL, Krogstad O. The effect of a partial bite raising splint on the occlusal face height. An xray cephalometric study in human adults. Acta Odontol Scand. 1982;40(1):17-24.

5. Reis A, Higashi C, Loguercio AD. Reanatomization of anterior eroded teeth by stratification with direct composite resin. J Esthet Restor Dent. 2009;21(5):304-16.

6. Dietschi D, Argente A. A comprehensive and conservative approach for the restoration of abrasion and erosion. part II: clinical procedures and case report. Eur J Esthet Dent. 2011;6(2):142-59.

7. Kalaykova SI, Sterenborg BAMM, Loomans BAC, Huysmans MDNJM. Impact of restorative treatment of tooth wear upon masticatory performance. J Dent. 2019;88:103159.

8. Bartlett D, Sundaram G. An up to 3-year randomized clinical study comparing indirect and direct resin composites used to restore worn posterior teeth. Int $\mathrm{J}$ Prosthodont. 2006;19(6):613-17.

9. Güth JF, Almeida E Silva JS, Ramberger M, Beuer F, Edelhoff D. Treatment concept with CAD/CAM-fabricated high-density polymer temporary restorations. J Esthet Restor Dent. 2012;24(5):310-18.

10. Hamburger JT, Opdam NJ, Bronkhorst EM, Kreulen CM, Roeters JJ, Huysmans MC. Clinical performance of direct composite restorations for treatment of severe tooth wear. J Adhes Dent. 2011;13(6):585-93

11. Johansson A, Johansson AK, Omar R, Carlsson GE. Rehabilitation of the worn dentition. J Oral Rehabil. 2008;35(7):548-66.

12. Opdam N, Skupien JA, Kreulen CM, Roeters J, Loomans B, Huysmans MD. Case Report: A Predictable Technique to Establish Occlusal Contact in Extensive Direct Composite Resin Restorations: The DSO-Technique. Oper Dent. 2016;41(S7):S96-S108.
13. Vailati F, Vaglio G, Belser UC. Full-mouth minimally invasive adhesive rehabilitation to treat severe dental erosion: a case report. J Adhes Dent. 2012;14(1):83-92.

14. Ramseyer ST, Helbling C, Lussi A. Posterior Vertical Bite Reconstructions of Erosively Worn Dentitions and the "Stamp Technique" - A Case Series with a Mean Observation Time of 40 Months. J Adhes Dent. 2015;17(3):283-89.

15. Perrin P, Zimmerli B, Jacky D, Lussi A, Helbling C, Ramseyer S. Die Stempeltechnik für direkte Kompositversorgungen [The stamp technique for direct composite restoration]. Schweiz Monatsschr Zahnmed. 2013;123(2):111-29.

16. Silva LD, de Lima VN, Faverani LP, de Mendonça MR, Okamoto R, Pellizzer EP. Maxillary sinus lift surgery-with or without graft material? A systematic review. Int J Oral Maxillofac Surg. 2016;45(12):1570-76.

17. Rizzo NSP, da Cunha LF, Sotelo BV, Gonzaga CC, Correr GM, Gaião U. Esthetic Rehabilitation with Direct Composite Resin in a Patient with Amelogenesis Imperfecta: A 2-Year Follow-Up. Case Rep Dent. 2019;2019:8407025.

18. Cogulu D, Becerik S, Emingil G, Hart PS, Hart TC. Oral rehabilitation of a patient with amelogenesis imperfecta. Pediatr Dent. 2009;31(7):523-27.

19. Reis B de O, Brogin FF, Catelan A, Briso ALF, Santos $\mathrm{PH}$ dos. Avaliação de diferentes procedimentos de acabamento e polimento sobre a rugosidade de superfície de resina composta submetida à escovação com dentifrício. Arch Health Invest. 2017 6(11): 524-29

20. Sampaio CS, Pizarro PG, Atria PJ, Hirata R, Giannini M, Mahn E. Effect of Shortened LightCuring Modes on Bulk-Fill Resin Composites. Oper Dent. 2020;45(5):496-505.

\section{CONFLITO DE INTERESSES}

Os autores declaram não haver conflitos de interesse

\section{AUTOR PARA CORRESPONDÊNCIA}

\section{Keith Murieli Ferreira de Magalhães}

Department of Dental Materials and Prosthesis,

São Paulo State University (UNESP),

School of Dentistry

José Bonifacio Street n: 1193

16015-050 Aracatuba - SP, Brazil.

Telephone: +55 (18) 3636-3237

E-mail: keithmagalhaes96@gmail.com 